\title{
Effectiveness of N95 Facepiece Respirators in Filtering Aerosol Following Storage and Sterilization
}

\author{
Tzu-Hsien Lin ${ }^{1,2}$, Chun-Chieh Tseng ${ }^{3}$, Yu-Li Huang ${ }^{4}$, Hsiu-Chuan Lin ${ }^{5,6}$, Chane-Yu Lai ${ }^{6,7 *}$, \\ Shu-An Lee (** $^{* *}$
}

${ }^{1}$ Department of Public Health, China Medical University, Taichung 40402, Taiwan

${ }^{2}$ Department of Occupational Safety and Health, China Medical University, Taichung 40402, Taiwan

${ }^{3}$ Department of Public Health, Tzu Chi University, Hualien 97004, Taiwan.

${ }^{4}$ Department of Safety, Health and Environmental Engineering, National Kaohsiung University of Science and

Technology, Kaohsiung 81164, Taiwan

${ }^{5}$ Centers for Disease Control, Taichung 40855, Taiwan

${ }^{6}$ Department of Occupational Safety and Health, Chung Shan Medical University, Taichung 40201, Taiwan

${ }^{7}$ Department of Occupational Medicine, Chung Shan Medical University Hospital, Taichung 40201, Taiwan.

${ }^{8}$ Department of Environmental Engineering and Science, Feng Chia University, Taichung 40724, Taiwan

\begin{abstract}
The use of electret N95 filtering facepiece respirators (N95FFRs) after prolonged storage or treatment can reduce the expense of buying non-expired N95 and thus enable developing and developed countries to optimize their use of limited resources to against airborne particles and diseases, such as coronavirus disease 2019 (COVID-19). The filtration performance of five N95FFR models following long-term storage, removal of charge using isopropanol alcohol (IPA), autoclaving, or treatment with gamma-radiation was assessed using a TSI 8130 automated filter tester. Statistically significant differences were found in the penetration $(P)$, pressure drop $(\Delta p)$ and quality factor $\left(q_{f}\right)$ between non-expired and expired N95 models 3M-8210 and 3M-8511. A statistically significant linear correlation was also obtained between the N95 penetration ratio $(P R)$ and the difference between year of manufacture and time of test $(D Y M)$. The $P R$ of N95 was more strongly influenced by eliminating the charge (for extremely out-of-date respirators) on the electret filter than by the $D Y M$. Sterilization by gamma irradiation increased the $P$ into non-expired and expired N95FFR models $(p<0.05)$, reducing their $q_{f}$. The $q_{f}$ of all N95FFR models, except UVEX-3200, was strongly affected by gamma irradiation, the removal of charge using IPA, autoclaving, and storage in that order. All expired models maintained acceptable filtration performance and still could be used to collect aerosol effectively, even though models 3M-8511 and 3M-1860 had been manufactured up to 13 years previously. As the COVID-19 outbreaks in 2019 and is getting worse in 2020, these data are useful in developing a global stockpiling strategy to maximize the longevity of N95FFRs for public health and healthcare workers. However, the aging of the straps and seal materials (rubbers, plastics) of the N95FFRs may affect their fit factor and effectiveness.
\end{abstract}

Keywords: N95; Storage; COVID-19; Autoclave; Gamma irradiation; Filtration performance.

\section{INTRODUCTION}

To protect environmental health, occupational hygiene, and the health of healthcare workers (HCWs) and home healthcare workers (HHWs) (Ollier et al., 2019), certified respiratory protection devices are used to prevent respiratory infections. Research reveals that patients can release airborne transmission-available pathogen particles and may be possible

\footnotetext{
* Corresponding author.

E-mail address: cylai511@gmail.com (C.Y. Lai); salee@fcu.edu.tw (S.A. Lee)
}

spread to a distance $>3 \mathrm{~m}$ from a patient with a respiratory disease (Lee et al., 2019). Respiratory protection devices are the last line of defense against such infections. Wearing respiratory protection devices is the most effective way to reduce exposure to aerosol, and a properly fitting N95 filtering facepiece respirator (N95FFR) is approximately 20 times more efficient than a surgical mask (Ollier et al., 2019).

During a severe acute respiratory syndrome (SARS), Middle East respiratory syndrome (MERS) or coronavirus disease 2019 (COVID-19) outbreak that became a national emergency, N95FFRs were in short supply (Leung et al., 2020). Health authorities in Taiwan began stockpiling N95FFRs for their HCWs after SARS event (Roberge, 2008; Chen et al., 2017). When many unused N95FFRs have reached 
their expiration dates, they were destroyed (Chen et al., 2017). In 2011, the Australian government developed a range of strategies to improve the efficiency of stockpile management (ANAO, 2014). One of them was the development of a fully costed model for extending the shelf-life of $\mathrm{P} 2$ respirators, involving an acceptable level of risk, to deliver significant savings, given that the items dominate the total capital cost of such a stockpile (ANAO, 2014).

The filtration efficiency of N95FFRs, including aerosol penetration $(P)$, pressure drop $(\Delta p)$ and filter quality $\left(q_{f}\right)$, is important in determining whether the risk of their use is acceptable (Lin et al., 2017a, b). Viscusi et al. (2009) investigated 21 types of NIOSH-certified N95FFR for use during flu pandemics. These respirators were manufactured from 1999 to 2001 and were stored for at least six years at temperatures between $15^{\circ} \mathrm{C}$ and $32^{\circ} \mathrm{C}$ and a relative humidity (RH) between $20 \%$ and $80 \%$. $\mathrm{NaCl}$ particles, certified by NIOSH, were used to measure $P$ at a test gas flow rate of $85 \mathrm{~L} \mathrm{~min}^{-1}$. The results thus obtained revealed that two of the 21 types of respirator had mean values of $P$ of greater than $5 \%$, and initial mean values from $0.39 \%$ to $5.83 \%$. Upon loading, the mean maximum values fell to between $0.95 \%$ and $5.83 \%$, revealing that the duration of storage was not directly related to whether the respirators met standards of filter performance (Viscusi et al., 2009). The relationship between the penetration and the year of manufacture of nonexpired/expired N95FFRs has not yet been accurately determined.

The contamination of N95FFRs during manufacture, transportation and prolonged storage is a concern. Common methods for decontaminating medical products involve an autoclave (steam sterilization) or gamma irradiation for large-scale sterilization (World Health Organization, 2004). The latter method has been approved by the WHO. The decontamination efficiency depends on the gamma irradiation dosage (da Silva Aquino, 2012). Exposure to gamma irradiation doses of less than $10 \mathrm{kGy}$ effectively deactivates pathogenic microorganisms such as Salmonella and Campylobacter. Irradiation doses of between 20 and $30 \mathrm{kGy}$ are most frequently used to treat food that is intended for consumption by pathogen-free animals (da Silva Aquino, 2012). The ISO (2013) recommends a sterilizing dose of 25 kGy for single-use medical devices. Therefore, an autoclave under general operating conditions with 10, 25, and $30 \mathrm{kGy}$ gamma irradiation is used for decontamination treatment herein.

The Taiwan CDC requires that for the storage of a stockpile of N95FFRs, the room temperature must not exceed $35^{\circ} \mathrm{C}$ and the relative humidity $(\mathrm{RH})$ is less than $80 \%$, or the storage conditions must be as recommended by the suppliers (Chen et al., 2017). Items must be placed on shelves, cabinets or pallets. All items must be stored by category and clearly labeled. Records of requisition, receipt and any damage, including wear and tear, must be maintained. All stockpiled N95FFRs must be dry, clean, undamaged, odorless and without exposed threads. The non-expired/expired N95FFR samples that were tested in this work had been stored under these conditions.

In accordance with US 42 CFR 84, this work compares the $P$ and $\Delta p$ values of disposable N95FFRs with various life expectancies to evaluate their $q_{f}$. To evaluate the effect of this factor on the filtering characteristics of electret N95FFRs, the $q_{f}$ of N95FFRs was also obtained before and after autoclave sterilization or gamma irradiation.

\section{MATERIAL AND METHODS}

\section{Collecting and Testing N95FFRs (Non-expired/Expired/ Extremely Out-of-date)}

This work involved five NIOSH-certified N95FFR models (Table 1), and they are 3M-8210, 3M-1860, SH-2950, 3M8511 and UVEX-3200 models. All these five N95FFR models are Taiwan stockpiling respirators after SARS event. $3 \mathrm{M}-8210$ model is a cup style mask with welded dual point attachment and has cushioning nose foam. 3M-1860 model is a cup shape mask with fluid resistant, braided headbands, and has cushioning nose foam. SH-2950 model is a flat-fold type mask with embossed fringe seal, and has an adjustable nose pad. 3M-8511 model is also a cup shape mask with braided headbands two-strap design, and has dual point attachment. UVEX-3200 model is a folding and cup-shaped mask with seamless headband and has an adjustable nose clip. The non-expired N95FFRs were purchased during the test and the out-of-date (expired) ones had been stored as part of Taiwan's stockpile program (Chen et al., 2017), enabling 'before-and-after' comparisons to be made (Viscusi et al., 2009) (Fig. 1). To compare the effectiveness of charged electret N95FFRs with that of uncharged ones, randomly selected new 3M-8210 N95FFRs were sprayed with isopropanol alcohol (IPA) to eliminate the electret filter charge (Lehtimäki and Heinonen, 1994; Huang et al., 2013).

A TSI 8130 automated filter tester (TSI Inc., St. Paul, $\mathrm{MN}$ ) was used to measure the filtering percentage $P$ and $\Delta p$, consistent with the US 42 CFR 84 particulate respirator certification protocol (NIOSH, 2016). Accordingly, the test aerosol was sodium chloride whose particle size distribution was a count median diameter of $0.075 \pm 0.020 \mu \mathrm{m}$ (most penetrating particle size) with a geometric standard deviation (GSD) of not more than 1.86. The test aerosols were passed through a bipolar aerosol neutralizer (Model 8113, TSI Inc., St. Paul, MN), which neutralized test aerosols to the Boltzmann charge equilibrium (Chang et al., 2018; Lin et al., 2019; Jung et al., 2020). The $99 \%$ test aerosol size range was from 0.007 to $0.882 \mu \mathrm{m}$. Before a filter was tested, it was placed in an environment of $85 \pm 5 \% \mathrm{RH}$ at $38 \pm 2.5^{\circ} \mathrm{C}$ for $25 \pm 1$ hours (NIOSH, 2016). Consistent with the testing standard US 42 CFR 84, the test was conducted in the next three minutes and the results were compared to those of an initial penetration test that were taken from another work (Viscusi et al., 2009). The initial penetration test can be used to determine the status of stored N95FFRs in preference to the more complex and longer maximum penetration test, which requires the respirator to be loaded with $200 \mathrm{mg}$ of sodium chloride aerosol (Viscusi et al., 2009). In this work, each respirator underwent an initial penetration test and six samples of each non-expired and expired model, for a total of 192 samples, were tested. 


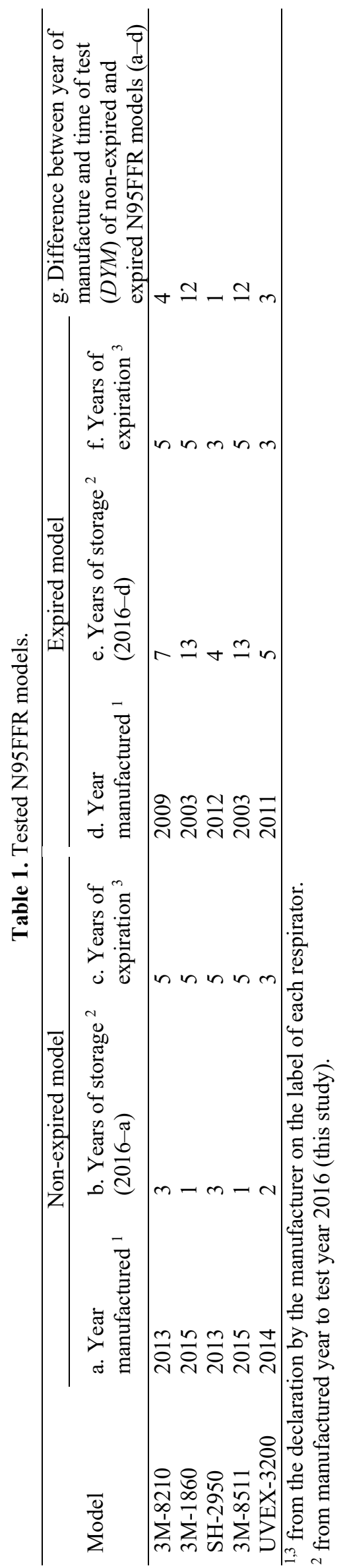

\section{Sterilization of N95FFRs}

The N95FFRs were sterilized using an autoclave (six respirators of each non-expired and expired model) or gamma irradiation (three respirators at each irradiation dose). They were heated for $15 \mathrm{~min}$ at $121^{\circ} \mathrm{C}$ under $1.06 \mathrm{~kg} \mathrm{~cm}^{-2}$ (15 pounds per square inch, psi) of pressure in an autoclave (Lin et al., 2018). In the irradiation test, the N95FFRs were subjected to integral irradiation doses $(\mathrm{hE} \gamma \mathrm{i}=1.17$ and $1.33 \mathrm{MeV},{ }^{60} \mathrm{Co}$ source) of 10,25 or $30 \mathrm{kGy}$ accumulated in air at a dose rate of $1.3 \mathrm{kGy} \mathrm{h}^{-1}$ (Viraneva et al., 2015). Then, they were tested using a TSI 8130 automated filter tester following the US 42 CFR 84 protocol (NIOSH, 2016).

\section{Calculation of Filter Quality and Penetration Ratio}

The $q_{f}$ of a filter incorporates $P$ and $\Delta p$, and is an index of filter performance (Huang et al., 2013). It is defined as follows.

$q_{f}=\frac{\ln \left(\frac{1}{p}\right)}{\Delta p}$

Both $P$ and $\Delta p$ should be considered in ranking N95FFRs. A lower $P$ and $\Delta p$ yield a higher $q_{f}$, indicating better filter performance (Lin et al., 2017a, b). When the penetration limit is $5 \%,\left(q_{f}\right.$ is 0.084 and $0.120 \mathrm{~mm} \mathrm{H}_{2} \mathrm{O}^{-1}$ when the inhalation and exhalation resistance limit is 35 and $25 \mathrm{~mm}$ $\mathrm{H}_{2} \mathrm{O}$, respectively (NIOSH, 2016). Since the test performance index of a single filter of US 42 CFR 84 has two values and uses two different limits (inhalation and exhalation resistance). In this study, these values will be used as standard references of $P, \Delta p$ and $q_{f}$.

To evaluate the amplitude of the $P$ of N95FFRs that were out of date and to standardize the measured performance of N95FFR models, the penetration ratio $(P R)$ is calculated as follows (Fig. 1).

$P R=\frac{P_{\text {expired }}}{P_{\text {non-expired }}}$

where $P_{\text {expired }}=$ particle penetration through an expired N95FFR, and $P_{\text {non-expired }}=$ particle penetration through a nonexpired N95FFR.

$P R=1$ means that particle penetration through an expired N95FFR that equals that through a non-expired one of the same model. $P R>1$ or $<1$ indicates that particle penetration through the expired N95FFR is higher or lower, respectively, than that through the non-expired one.

\section{Statistical Analysis}

For each of the five tested N95FFR models (six or three samples of each model under each test condition), the mean and standard deviation of $P R$ were calculated in Microsoft Excel (Microsoft Corp, Redmond, WA). Each mean $P$ was categorized as either passing or failing with respect to the maximum allowable penetration level of $5 \%$ for NIOSH certification (Viscusi et al., 2009). The 'before-and-after' comparisons were made using non-parametric statistical tools 


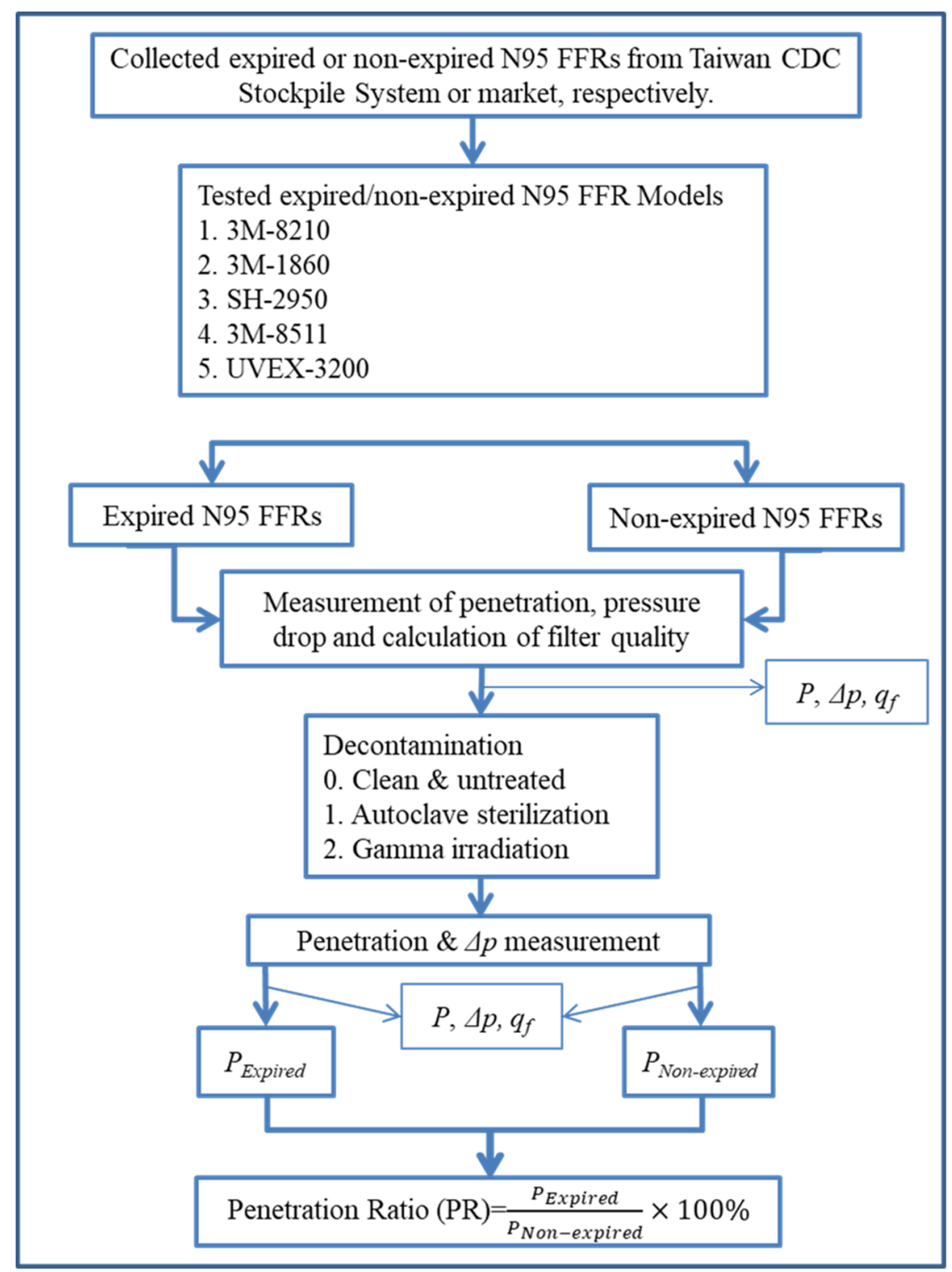

Fig. 1. Experimental flow-chart.

in SPSS software, version 17.0 (SPSS Inc., Chicago, IL). Two regression models, linear and logarithmic, were used to determine the relationship between $P R$ and the difference between year of manufacture and time of test $(D Y M)$ of the non-expired and expired N95FFRs. Statistical differences were considered to be significant at $p<0.05$.

\section{RESULTS}

\section{Effect of Storage Duration on Performance of N95FFRs}

The $P$ and $\Delta p$ of all expired and non-expired N95FFRs met the US 42 CFR 84 standards $(P<5 \%$ in Fig. 2 and $\Delta p<$ $25 \mathrm{~mm} \mathrm{H}_{2} \mathrm{O}$ in Fig. 3). All expired N95FFRs had a higher $P$ and a lower $\Delta p$ than the non-expired ones of the same model. In Fig. 2 , the $P$ of the expired $3 \mathrm{M}-8210$ is $0.399 \%( \pm 0.218)$ and the non-expired one is $0.212 \%( \pm 0.087 \%)$. The $P$ of the expired 3M-1860, SH-2950, 3M-8511 and UVEX-3200 is $0.924( \pm 0.558), 0.617( \pm 0.265), 1.586( \pm 0.302)$, and 4.772
( \pm 3.737$) \%$, respectively. Moreover, the $P$ of the non-expired $3 \mathrm{M}-1860$, SH-2950, 3M-8511 and UVEX-3200 is 0.383 $( \pm 0.122), 0.549( \pm 0.042), 0.568( \pm 0.099)$, and 2.490 $( \pm 1.194) \%$, individually. All these five N95FFR models had passed the US 42 CFR 84 N95 penetration criteria. However, expired and non-expired UVEX-3200 revealed higher standard error on $P$ than the other four N95FFR models.

Fig. 3 indicated that all the expired N95FFR models had lower $\Delta p$ than the non-expired models. Besides, all the expired or non-expired N95FFR models approved the US 42 CFR 84 criteria on exhalation or inhalation resistance. The $\Delta p$ of expired 3M-8210, 3M-1860, SH-2950, 3M-8511 and UVEX-3200 is $7.794( \pm 0.439), 9.056( \pm 1.126), 10.161$ $( \pm 1.933), 8.511( \pm 0.143)$ and $11.133( \pm 1.048) \mathrm{mmH}_{2} \mathrm{O}$, respectively. Additionally, the $\Delta p$ of non-expired $3 \mathrm{M}-8210$, $3 \mathrm{M}-1860$, SH-2950, 3M-8511 and UVEX-3200 is 8.778 $( \pm 0.435), 9.639( \pm 0.607), 10.994( \pm 0.362), 9.333( \pm 0.392)$ and $11.550( \pm 0.650) \mathrm{mmH}_{2} \mathrm{O}$, respectively. 


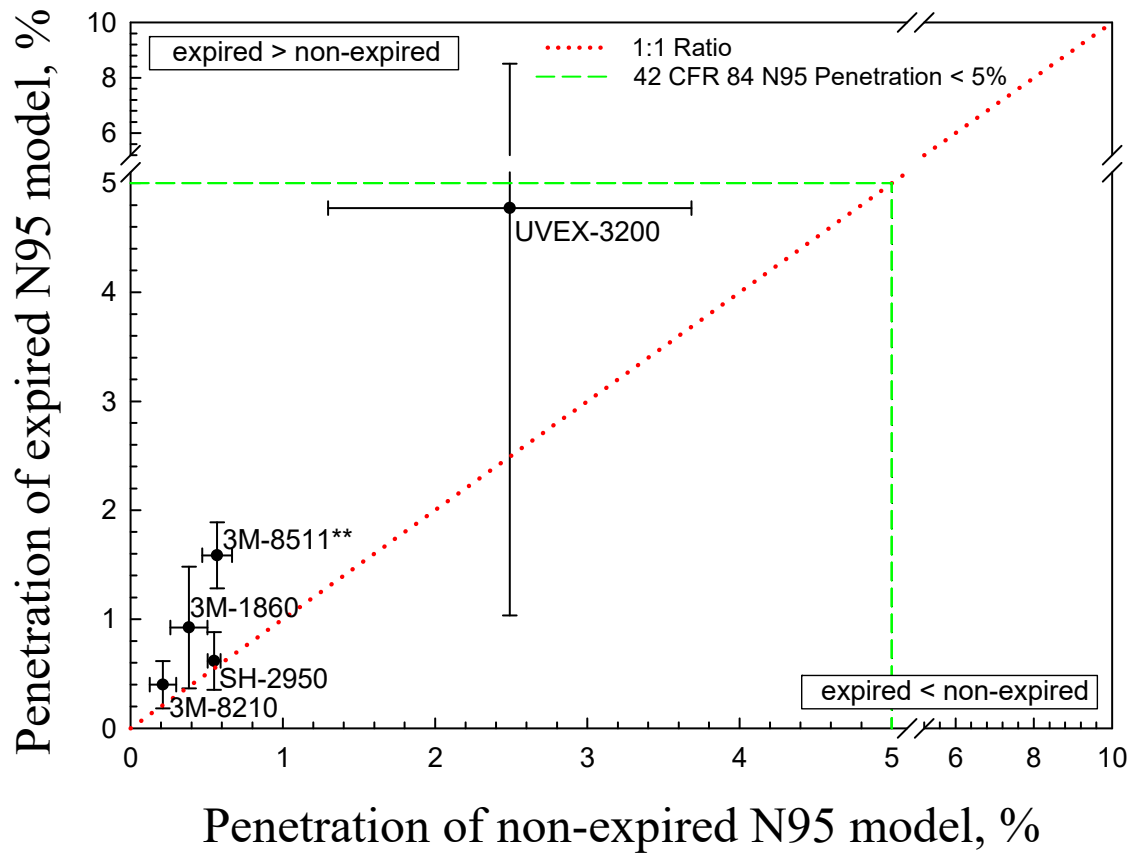

Fig. 2. Penetration of particles through non-expired and expired N95FFR models. Error bars represent one standard deviation.

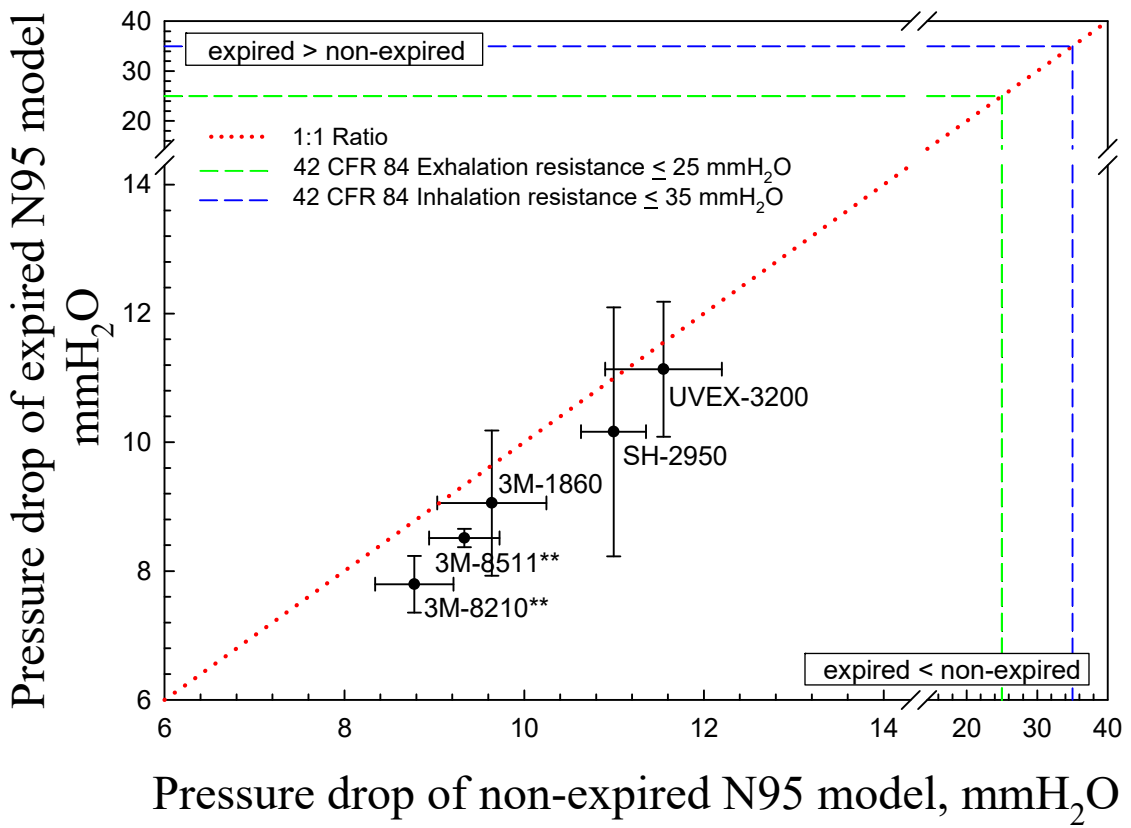

Fig. 3. Pressure drop across non-expired and expired N95FFR models. Error bars represent one standard deviation.

All five N95FFR models had better $q_{f}$ than US 42 CFR 84 breathing and penetration criteria with reference to Eq. 1, as shown in Fig. 4. The $q_{f}$ of expired 3M-8210, 3M-1860, SH$2950,3 \mathrm{M}-8511$ and UVEX-3200 is $0.728( \pm 0.102), 0.551$ ( \pm 0.147$), 0.524( \pm 0.107), 0.489( \pm 0.019)$ and $0.300( \pm 0.077)$, respectively. Also, the $q_{f}$ of non-expired 3M-8210, 3M-1860, SH-2950, 3M-8511 and UVEX-3200 is $0.711( \pm 0.067), 0.585$ $( \pm 0.058), 0.474( \pm 0.018), 0.556( \pm 0.027)$ and $0.330( \pm 0.053)$ correspondingly. No statistically significant difference in $\mathrm{P}, \Delta p$ or $q_{f}$ existed between the expired and non-expired N95FFR models, except for the $\Delta p$ of model 3M-8210 (Fig. 3) and the $P, \Delta p$ and $q_{f}$ of model 3M-8511 (Figs. 2, 3, and 4).

Fig. 5 plots the relationship between $P R$ and $D Y M$ for the non-expired and expired N95FFR models. As DYM increased from one to 12 years, $P R$ increased from 1.1 to as high as 2.8 (model 3M-8511), as calculated using Eq. (2). The values of $\mathrm{R}^{2}$ that were obtained using both the linear and the logarithmic regression models exceeded 0.8 . When randomly selected new model 3M-8210 N95FFRs were sprayed with IPA, their $P R$ was 69 (Eq. (2), 14.49 divided by $0.21,3 \mathrm{M}$ 8210 ' in Fig. 5) when the DYM was assumed to be infinite $(\infty)$. Calculations made using the linear regression or logarithmic 


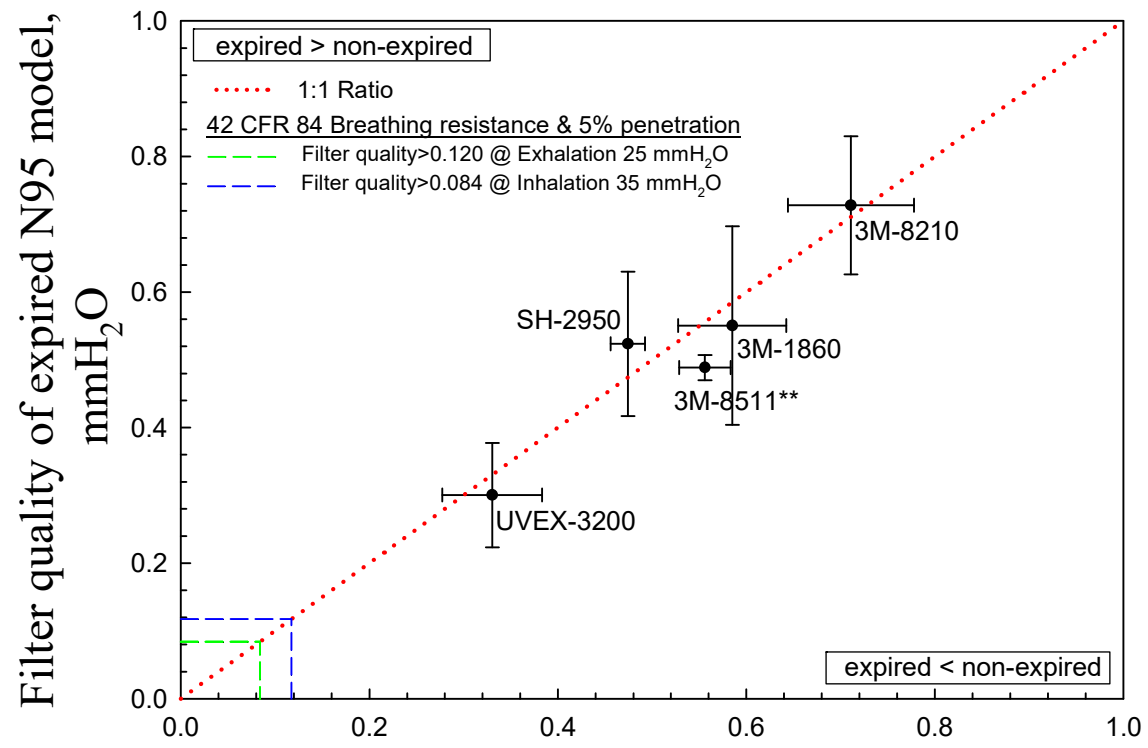

Filter quality of non-expired $\mathrm{N} 95$ model, $\mathrm{mmH}_{2} \mathrm{O}$

Fig. 4. Filter quality of non-expired and expired N95FFR models. Error bars represent one standard deviation.

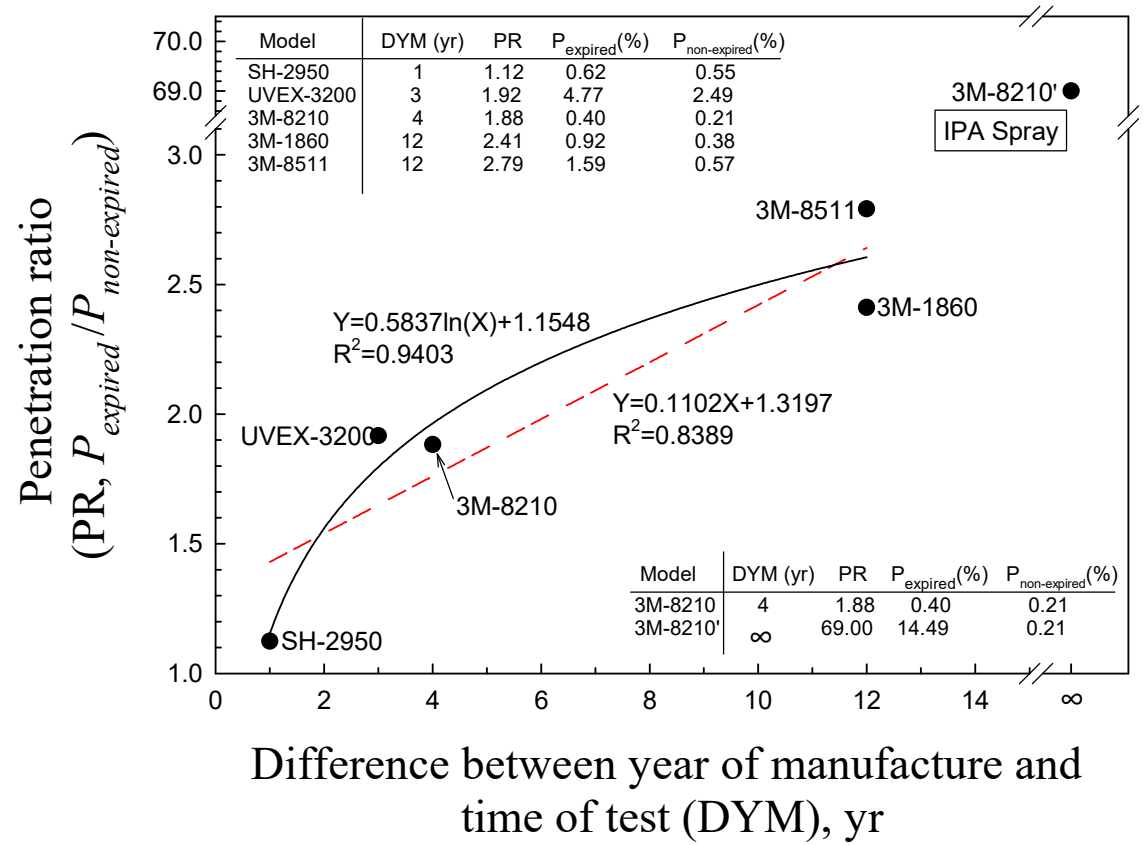

Fig. 5. Penetration ratio of non-expired and expired N95FFR models vs. difference between year of manufacture and time of test $(D Y M)$.

regression model yielded $P R=69$ at $D Y M=614$ or $3 \mathrm{E} 50$ years, respectively. Eq. (1) yielded a $q_{f}$ of 0.18 for $3 \mathrm{M}-8210$ '.

\section{Effect of Sterilization on Performance of N95FFR Models}

Treatment using an autoclave significantly increased the $P(p<0.05)$ only of model 3M-8511 (Fig. 6). Sterilization by gamma irradiation increased the $P$ of both non-expired and expired N95FFR models $(p<0.05)$. Sterilization using an autoclave caused the $P$ of two of the five expired N95FFR models to exceed the 5\% certification penetration limit (Lehtimäki and Heinonen, 1994). Sterilization by gamma irradiation yielded $P$ values of the five N95FFR models of 23 to $56 \%$. Treatment using an autoclave or gamma irradiation caused all N95FFR models, non-expired and expired, to satisfy the $25 \mathrm{~mm} \mathrm{H}_{2} \mathrm{O}$ certification breathing resistance requirement of US 42 CFR 84 (NIOSH, 2016) (Fig. 7).

Sterilization using an autoclave or gamma irradiation reduced the $q_{f}$ values of both non-expired and expired N95FFR models $(p<0.05)$ (Fig. 8). The $q_{f}$ values of models 3M-8210, 
$3 \mathrm{M}-1860$ and $3 \mathrm{M}-8511$ following gamma irradiation, as calculated using Eq. (1), did not satisfy the $0.084 \mathrm{~mm} \mathrm{H}_{2} \mathrm{O}^{-1}$ certification criterion. No statistically significant variation in $P, \Delta p$ or $q_{f}$ with gamma dose was observed.

\section{DISCUSSION}

Consistent with US 42 CFR 84 (NIOSH, 2016), variation of the $q_{f}$, including $P$ and $\Delta p$, of the N95FFR models with

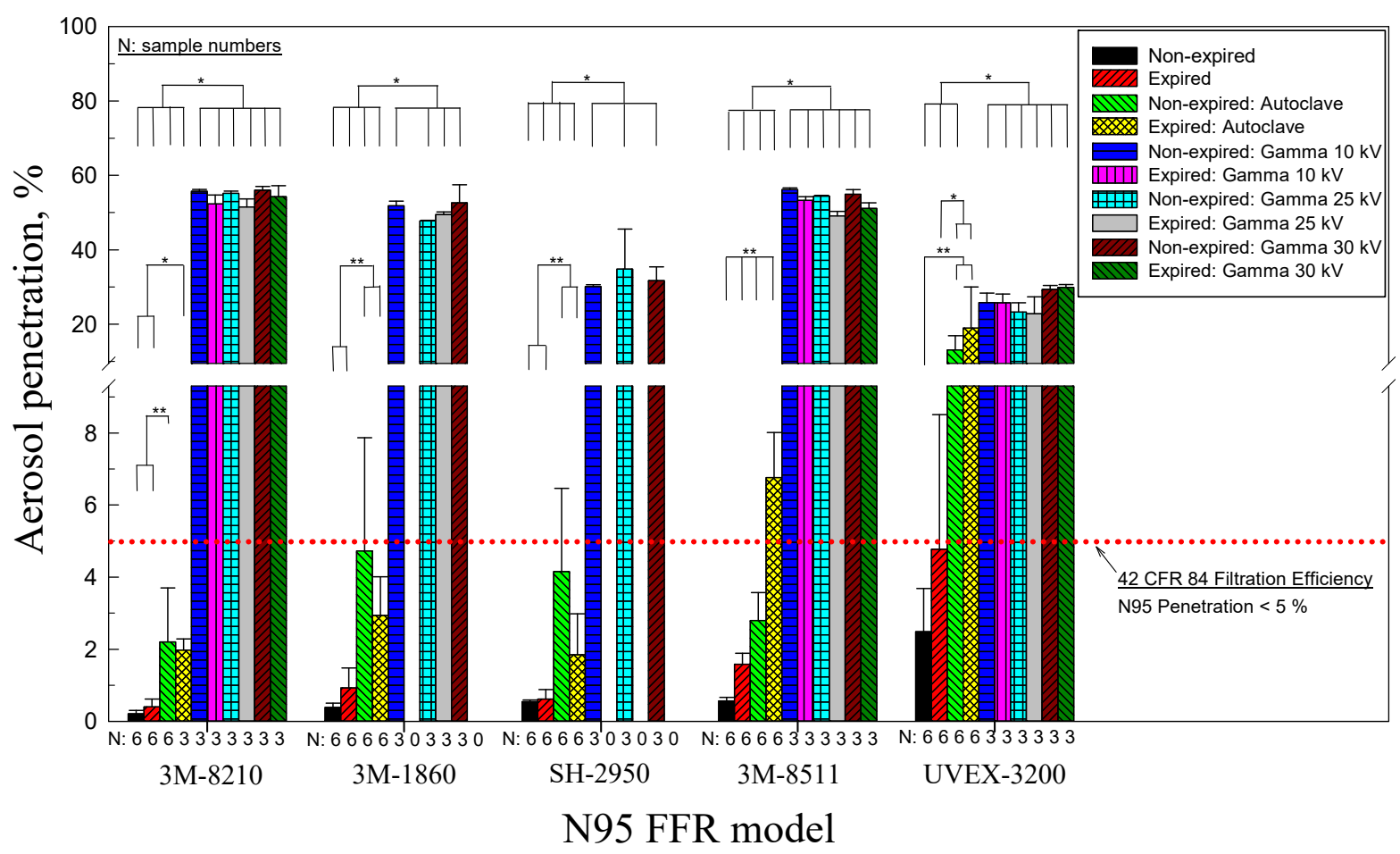

Fig. 6. Penetration of particles through non-expired and expired N95FFR models following sterilization. Error bars represent one standard deviation. "N" is number of samples.

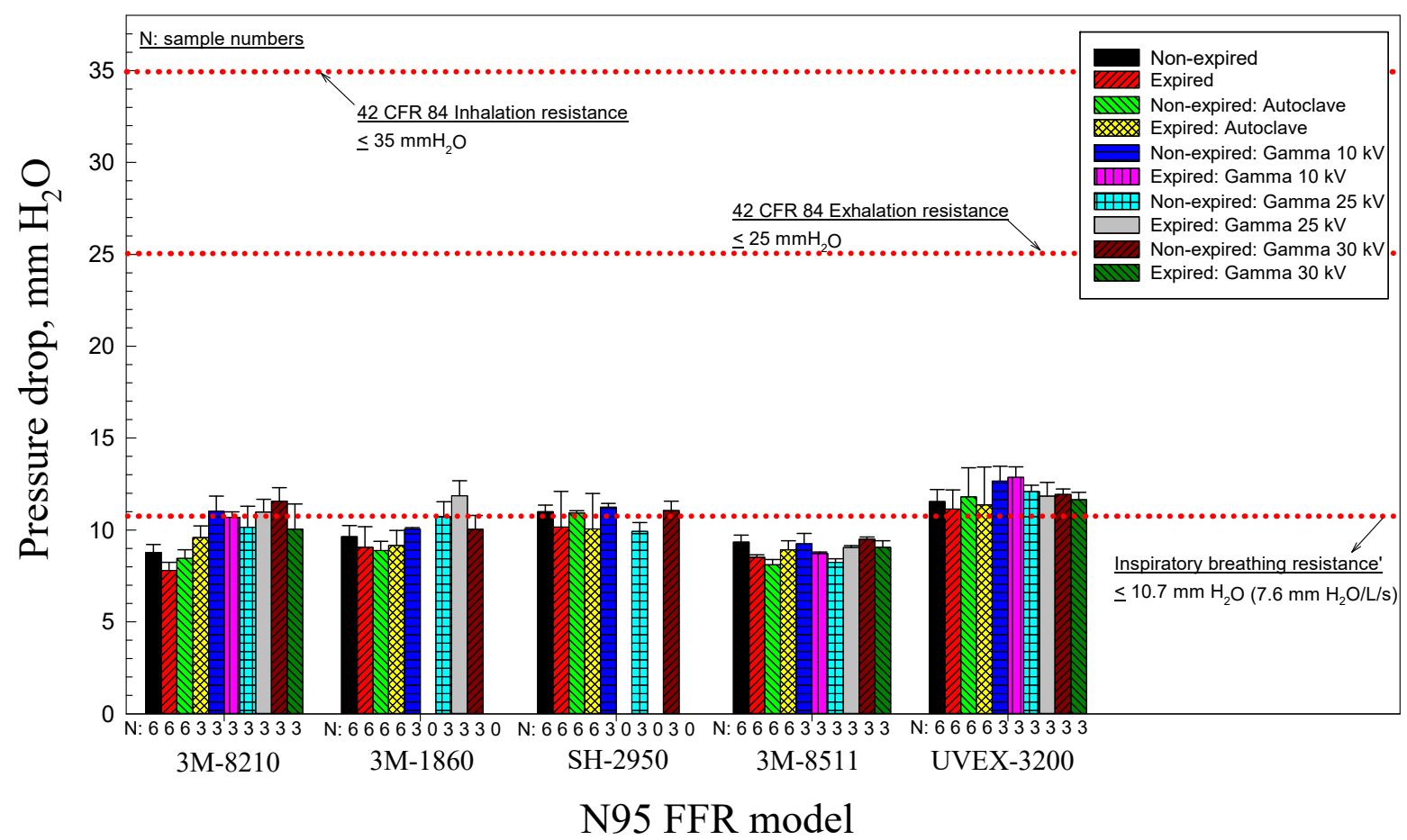

Fig. 7. Pressure drop across non-expired and expired N95FFR models following sterilization. Error bars represent one standard deviation. "N" is number of samples. 


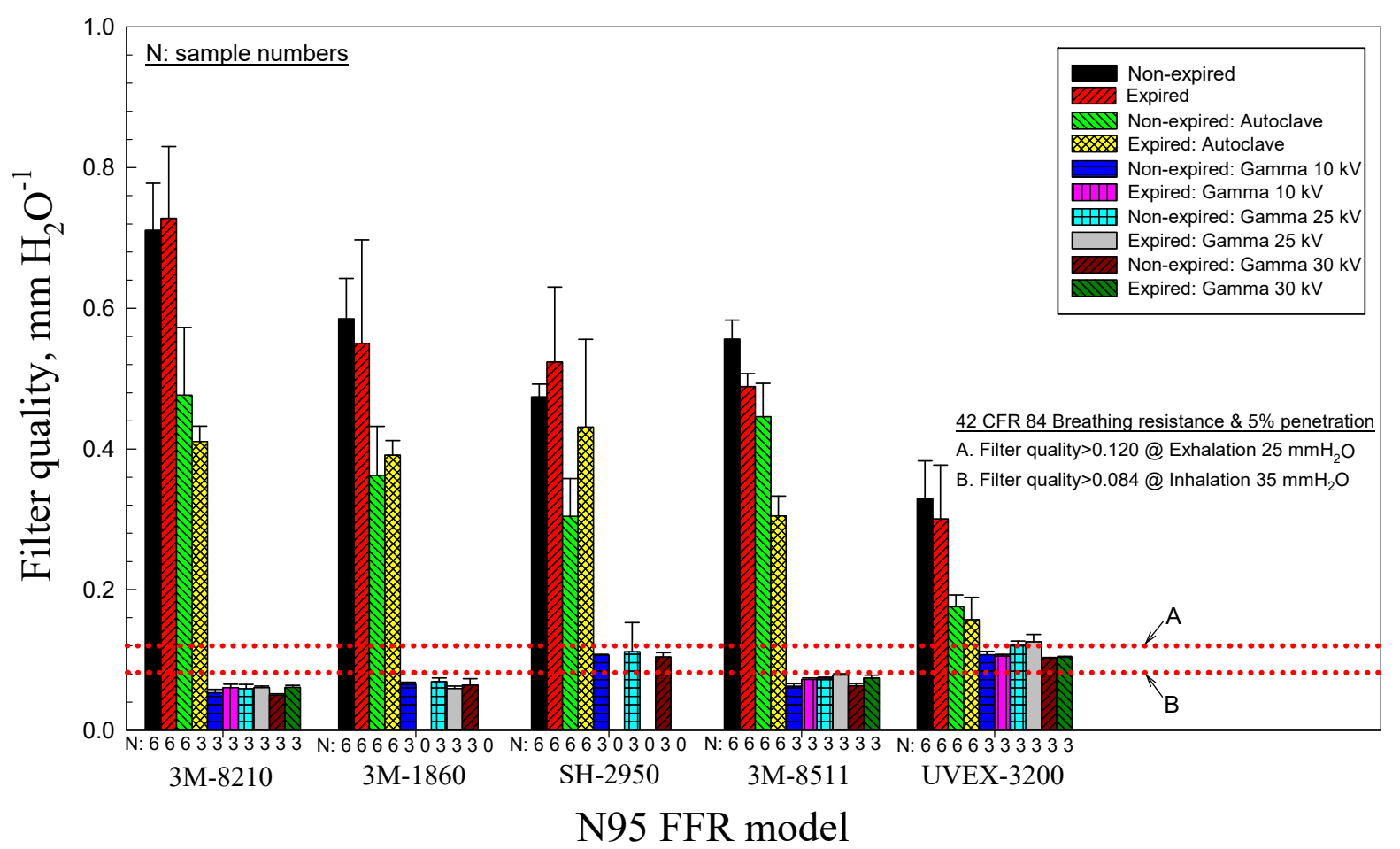

Fig. 8. Filter quality of non-expired and expired N95FFR models following sterilization. Error bars represent one standard deviation. " $\mathrm{N}$ " is number of samples.

the duration of validity is considered. Although all mean $P$ values of the expired N95FFR models exceeded those of the non-expired N95FFR models, the difference was only significant $(p<0.01)$ for model 3M-8511 (Fig. 2). Most of the expired N95FFR models maintained an acceptable $P$ even 13 years after their manufacture (Table 1). The $\Delta p$ values of the expired N95FFR models were lower than those of non-expired models (Fig. 3). The $\Delta p$ values of non-expired and expired N95FFR of models 3M-8210 also differed significantly, as did those of 3M-8511 $(p<0.01)$. All expired N95FFR models had $\Delta p$ values that satisfied the requirement of US 42 CFR 84 and were better than those of non-expired N95FFR models. Only the $q_{f}$ values of the non-expired and expired 3M-8511 N95FFR differed significantly $(p<0.01)$, and the mean $q_{f}$ values of the expired N95FFR models (were close to the 1:1 ratio line, or $\mathrm{x}=\mathrm{y}$ line) (Fig. 4). All of the expired N95FFR models had acceptable $q_{f}$ values.

An earlier study found no relationship between the duration of storage and failure to pass the initial penetration test and it found that the degree of degradation of filtration efficiency was probably model-specific (Viscusi et al., 2009). These discrepancies could have arisen from a lack of dates on the original packaging of 15 of the 21 models in that earlier work. In this work, no relationship between $P$ and the duration of storage between one and 13 years was found. Since the degree of filtration efficiency degradation depended on the model, two novel parameters, $P R$ and $D Y M$, were developed and used herein, and two statistically significant regressions of these parameters were obtained (Fig. 5).

For the linear regression, $\mathrm{R}^{2}$ was 0.839 , while for the logarithmic regression, it was slightly higher at 0.940 (Fig. 5).
Both regression analyses revealed that the $P R$ approached 2.6 at a $D Y M$ of 12 , which was the upper limit of each regression. Both regression models provided a positive correlation between $P R$ and $D Y M$. To predict the highest possible $P R$ value, non-expired model 3M-8210 N95FFRs were sprayed with IPA to eliminate the charge on the electret filter (Lehtimäki and Heinonen, 1994; Huang, et al., 2013). The $P R$ of both the sprayed and unsprayed model 3M-8210 N95FFRs was 69 (as shown in Fig. 5), revealing that the linear regression may be more effective than the logarithmic regression in providing the relationship between $P R$ and $D Y M$. However, this suggestion warrants further study.

The reference model herein was the non-expired and predecontaminated N95FFR model. Decontamination using either an autoclave or gamma irradiation statistically significantly increased the $P$ of all non-expired and expired N95FFR models (Fig. 6). The autoclaved expired models 3M-8210, $3 \mathrm{M}-1860$ and SH-2950 still met the 5\% penetration criterion for certification. However, gamma irradiation caused all N95FFR models to violate the $5 \%$ penetration criterion. Lin et al. (2017) used five decontamination methods to retrieve N95FFRs, and revealed decontamination increased the sizes of the most penetrating particles, changing the $q_{f}$ values of all of the N95FFRs. Moreover, chemical decontamination methods (bleach, IPA and ethanol) reduced the overall filter quality values for the three electret N95FFRs. On the other hand, physical decontamination methods (Taiwan traditional rice cooker and autoclave) are less destructive to the N95FFRs than chemical methods, and recommended that when buying new or reclaiming N95FFRs, penetration efficiency should be considered as the priority. 
The inhalation resistance of none of the tested N95FFR models came close to the US 42 CFR 84 specification (Fig. 7). However, the previously reported the range of normal threshold for the detection of inspiratory breathing resistance (IBR) was 6-7.6 $\mathrm{mm} \mathrm{H}_{2} \mathrm{O}\left(\mathrm{L} \mathrm{s}^{-1}\right)^{-1}$ (Roberge et al., 2013). The calculated IBR at $85 \mathrm{~L} \mathrm{~min}^{-1}$ was $10.7 \mathrm{~mm} \mathrm{H}_{2} \mathrm{O}$ (Fig. 7). Two of the five pre-decontaminated N95FFR models (SH2950 and UVEX-3200) did not meet the IBR threshold. Similar results were obtained following use of an autoclave. Gamma irradiation caused four of the five models to exceed the IBR threshold but not model 3M-8511. The IBR was probably related to the normal breathing resistance threshold for HCWs (Roberge et al., 2013; Lin et al., 2017b).

Two of the five expired N95FFR models (3M-8210 and SH-2950) had better $q_{f}$ values than the corresponding nonexpired ones (Fig. 8) because they had better $\Delta p$ (Fig. 7). However, no significant statistical difference existed between either the $\Delta p$ values or the $q_{f}$ values of the non-expired and expired models. No significant difference in the $P, \Delta p$ or $q_{f}$ values between the non-expired and expired N95FFR models existed following the use of the autoclave or sterilization by gamma irradiation. Significant differences in the $P, \Delta p$ or $q_{f}$ values were observed as a result of decontamination of the five N95FFR models, and gamma irradiation yielded a statistically significantly higher $P$ and lower $q_{f}$ than the autoclave. Since all of the $\Delta p$ values were around the IBR threshold, filter penetration dominated $q_{f}$.

No statistically significant variation of $P, \Delta p$ or $q_{f}$ with the amount of gamma irradiation $(10,25$, and $30 \mathrm{kGy})$ was observed when the N95FFR models were decontaminated therewith, probably because the accumulated dose rates were all about $1.3 \mathrm{kGy} \mathrm{h}^{-1}$. Exposure to gamma irradiation might have had an effect similar to that of IPA, which eliminated the charge on the electret filter (Lehtimäki and Heinonen, 1994; Huang et al., 2013). In 1962, research from Gross and Moraes revealed that the electret charge can be released by reheating of the sample or accompanied by a depolarization current. The depolarization might be brought about by $\mathrm{x}$-ray or gamma irradiation. Gamma irradiation is a recognized method of sterilization (Rutala and Weber, 2008) but greatly increases $P$ of the N95FFRs.

The above results show that the filter performance of the N95FFRs that were stored for a long time (such as the expired N95FFR models) generally met the US 42 CFR 84 standards for $P$ and $\Delta p$. Therefore, the storage duration of 13 years, considered herein, was not an important factor in determining the charge characteristics or $q_{f}$ of the N95FFRs. The $q_{f}$ values of the four N95FFR models other than UVEX-3200 were strongly influenced by gamma radiation, the removal of charge by IPA ( $q_{f}$ of $\left.3 \mathrm{M}-8210^{\prime}=0.18\right)$, autoclaving, and storage in that order (Fig. 8). Since enough expired N95FFR models could not be obtained and the test variables were many, the number of test samples (three or six) was lower than $(\mathrm{n}=20)$ required by US 42 CFR 84 (NIOSH, 2016).

All five N95FFR models, either non-expired or expired, had penetration levels of below $5 \%$. The filtration test that was used in this study was comparable to the NIOSH certification test and was performed under a set of "worstcase' conditions (high flow rate, charge-neutralized particles, and $85 \% \mathrm{RH}$ ). As stated above, 19 of the 21 models that were stored for up to ten years also had initial penetration levels of less than 5\% (Viscusi et al., 2009).

All five N95FFR models in this work met the standards for $P$ and $\Delta p$. Although one model (3M-8511) exhibited a significant increase in $P$ after the expiration date, the $P$ values of the other four models increased after expiration but not significantly. The $\Delta p$ values of all five expired N95FFR models were lower after the expiration date than before it; however, the change was significant for only two models (3M-8511 and 3M-8210). One model (3M-8511) had a significantly reduced $q_{f}$ while the other four models exhibited no significant change in $q_{f}$. The change in filter performance upon prolonged storage and sterilization was therefore model-specific (Mueller et al., 2018).

A related study by Viscusi et al. (2009) was not a typical aging study, and so did not offer true "before-and-after" comparisons. In contrast, the present study compares the filter performances of non-expired and expired N95FFR models. It is a more formal aging study that provides useful information on the potential longevity of stored N95FFRs for stockpile authorities. In this study, a true "'before-and-after' analysis was performed by performing a non-parametric paired t test to determine statistically significant changes in $P, \Delta p$ and $q_{f}$.

This study is limited by the fact that only five N95FFR models and 192 samples were analyzed, because other expired N95FFR models had been discontinued, consequently, nonexpired ones could not be purchased during the study. The other non-expired N95FFR models in the current Taiwan stockpile program do not have any expired counterparts. Notably, US 42 CFR 84 requires that all changes and modifications to N95FFRs are examined by NIOSH to determine whether testing is required (NIOSH, 2016). In this work, all results of tests of non-expired and expired N95FFR models met the NIOSH test standards. However, N95FFR manufacturers are continually modifying, refining, and restructuring their products to continue to satisfy NIOSH certification performance standards without always changing the model numbers (Viscusi et al., 2009). Therefore, the N95FFR models that were considered herein may or may not be representative of commercially available today.

The results in this investigation show that all five expired models (without sterilization) maintained a $P$ of below $5 \%$ and met the US 42 CFR 84 requirement. Therefore, this investigation is the first study to prove that these five expired models can still be used against aerosol even though two of them (3M-8511, 3M-1860) had been manufactured 13 years ago. Research study has suggested that respirator stockpile administrators should consider contacting respirator manufacturers to determine whether their products have recommended storage duration limits (Viscusi et al., 2009). Another study has recommended that stockpile authorities should consider periodic (perhaps annual) evaluations of representative samples from their stockpile to ensure that their N95FFRs maintain the required $P, \Delta p$ and $q_{f}$ after several years of storage (Viscusi et al., 2009; Lin et al., 2017a, b).

Shakya et al. (2017) showed that inexpensive cloth masks were extensively used in developing countries to protect against particulate pollution. However, inexpensive cloth 
masks are only marginally beneficial in protecting individuals from particles of size $<2.5 \mu \mathrm{m}$ while N95FFRs were found to be more effective in removing most tested particles (Shakya et al., 2017). If expired but usable N95FFRs must be worn by HCWs or other users, then stockpile authorities or users must consider that the aging of N95FFRs and the conditions thereof may affect their straps and seal materials (rubbers, plastics) and, therefore, their wearing fit factor and effectiveness (Viscusi et al., 2009; Lam et al., 2011; Spies et al., 2011; Lin et al., 2017a, b; Meadwell et al., 2019).

\section{CONCLUSIONS}

Following the stockpile program, prolonged storage duration did not strongly affect the $q_{f}$ of the NIOSH-certified N95FFR models in this study, although all five expired N95FFR models had a higher $P$ and a lower $\Delta p$ than the nonexpired ones of the same model. The mean aerosol $P$ and $\Delta p$ of all models remain met US 42 CFR 84 respirator test standards within and outside the period of validity. Without decontamination by gamma irradiation or the use of an autoclave, all expired N95FFR models maintained acceptable filtration performance and still could be used to collect aerosol effectively, even though models 3M-8511 and 3M-1860 had been manufactured up to 13 years formerly.

Ideally, N95FFRs should not be used after the manufacturer's stated expiration date. However, if a pandemic should strike and diseases control materials are in short supply, then authorities should consider extending the use of stockpiled and expired N95FFRs. Therefore, expired N95FFRs that nevertheless met the US 42 CFR 84 standards for filter performance can still be used in some situations. For example, extending their service lives following proper inspection and testing effectively reduces the cost of buying non-expired devices for protecting against airborne particles and accordingly enables countries to optimize their use of limited resources for training related to protection against airborne particles and disease prevention, especially when the world is suffering COVID-19 outbreak and N95FFRs are in short supplies. Before expired N95FFRs are used, testing data that are obtained by reliable testing institutions should be analyzed to ensure their effectiveness. Fit factor is still another important issue and should be test before one need to use the expired N95FFRs.

\section{DECLARATION}

\section{Availability of Data and Materials}

All data generated or analyzed in this study are included in the published article.

\section{COMPETING INTERESTS}

The authors declare no competing interests.

\section{FUNDING}

This work was supported by the Chung Shan Medical University joint research program with Feng Chia
University [FCU/CSMU 105-001]. The Taiwan Center for Disease Control [1031700321] provided a master's student stipend to Hsiu-Chuan Lin.

\section{ACKNOWLEDGEMENTS}

We thank the employees of China Biotech Corporation, Taiwan, for their assistance with the gamma irradiation tests. We are also grateful to the employees of OSH Co. Ltd., Taiwan, for making important contributions.

\section{REFERENCES}

Australian National Audit Office (ANAO) (2014). Management of the National Medical Stockpile. Department of Health. Australian National Audit Office. ANAO Audit Report No.53 2013-14, June 2014. https://www.anao.gov.au/sites/g/files/net616/f/AuditReport 2013-2014_53.pdf. Last Access: 28 January 2019.

Chang, D.Q., Liu, J.X. and Chen, S.C. (2018). Factors affecting particle depositions on electret filters used in residential HVAC systems and indoor air cleaners. Aerosol Air Qual. Res. 18: 3211-3219.

Chen, Y.J., Chiang, P.J., Cheng, Y.H., Huang, C.W., Kao, H.Y., Chang, C.K., Huang, H.M., Liu, P.Y., Wang, J.H., Chih, Y.C., Chou, S.M., Yang, C.H. and Chen, C.H. (2017). Stockpile model of personal protective equipment in Taiwan. Health Secur. 15: 170-174.

da Silva Aquino, K.A. (2012). Sterilization by gamma irradiation. Gamma Radiat. 9: 172-202.

Gross, B. and De Moraes, R.J. (1962). Gamma irradiation effects on electrets. Phys. Rev. 126: 930.

Huang, S.H., Chen, C.W., Kuo, Y.M., Lai, C.Y., McKay, R. and Chen, C.C. (2013). Factors affecting filter penetration and quality factor of particulate respirators. Aerosol Air Qual. Res. 13: 162-171.

ISO (2013) ISO 11137 - Sterilization of Health Care Products Package.

Jung, S., Gersten, B.T. and Biswas, P. (2020). Quantifying the effect of nonwoven conductive fabric liners on electrostatic precipitator submicrometer particle removal efficiency. Aerosol Air Qual. Res. 20: 489-498.

Lam, S.C., Lee, J.K.L., Yau, S.Y. and Charm, C.Y.C. (2011). Sensitivity and specificity of the user-seal-check in determining the fit of N95 respirators. J. Hospital Infect. 77: 252-256.

Lee, J., Yoo, D., Ryu, S., Ham, S., Lee, K., Yeo, M., Min, K. and Yoon, C. (2019). Quantity, size distribution, and characteristics of cough-generated aerosol produced by patients with an upper respiratory tract infection. Aerosol Air Qual. Res. 19: 840-853.

Lehtimäki, M. and Heinonen, K. (1994). Reliability of electret filters. Build. Environ. 29: 353-355.

Leung, C.C., Lam, T.H. and Cheng, K.K. (2020). Mass masking in the COVID-19 epidemic: People need guidance. Lancet 395: 945.

Lin, C.W., Huang, S.H., Chang, K.N., Kuo, Y.M., Wu, H.D., Lai, C.Y. and Chen, C.C. (2019). Experimental measurements of regional lung deposition in Taiwanese. 
Aerosol Air Qual. Res. 19: 832-839.

Lin, T.H., Chen, C.C., Huang, S.H., Kuo, C.W., Lai, C.Y. and Lin, W.Y. (2017a). Filter quality of electret masks in filtering 14.6-594 nm aerosol particles: Effects of five decontamination methods. PLoS One 12: e0186217.

Lin, T.H., Tang, F.C., Chiang, C.H., Chang, C.P. and Lai, C.Y. (2017b). Recovery of bacteria in filtering facepiece respirators and effects of artificial saliva/perspiration on bacterial survival and performance of respirators. Aerosol Air Qual. Res. 17: 187-197.

Lin, T.H., Tang, F.C., Hung, P.C., Hua, Z.C. and Lai, C.Y. (2018). Relative survival of Bacillus subtilis spores loaded on filtering facepiece respirators after five decontamination methods. Indoor Air 28: 754-762.

Meadwell, J., Paxman-Clarke, L., Terris, D. and Ford, P. (2019). In search of a performing seal: Rethinking the design of tight-fitting respiratory protective equipment facepieces for users with facial hair. Safety Health Work 10: 275-304.

Mueller, W., Horwell, C.J., Apsley, A., Steinle, S., McPherson, S., Cherrie, J.W. and Galea, K.S. (2018). The effectiveness of respiratory protection worn by communities to protect from volcanic ash inhalation. Part I: Filtration efficiency tests. Int. J. Hyg. Environ. Health 221: 967976.

National Institute of Occupational Safety and Health (NIOSH) (2016). Approval of respiratory protective devices: 42 C.F.R. part 84.180 and 84.181.

Ollier, K., Leppänen, M., Wu, B., Yermakov, M., Newman, N.C., Reponen, T. and Grinshpun, S.A. (2019). Inhalation exposure and respiratory protection of home healthcare workers administering aerosolized medications (simulation study). Aerosol Air Qual. Res. 19: 937-944. doi: 10.4209/ aaqr.2018.10.0366.

Roberge, R. (2008). Effect of surgical masks worn concurrently over N95 filtering facepiece respirators:
Extended service life versus increased user burden. $J$. Pub. Health Manage 14: E19-26.

Roberge, R.J., Kim, J.H., Powell, J.B., Shaffer, R.E., Ylitalo, C.M. and Sebastian, J.M. (2013). Impact of low filter resistances on subjective and physiological responses to filtering facepiece respirators. PLoS One 8: e84901. doi: 10.1371/journal.pone.0084901.

Rutala, W.A., Weber, D.J. and the Healthcare Infection Control Practices Advisory Committee (HICPAC) (2008). Guideline for Disinfection and Sterilization in Healthcare Facilities, 2008. Centers for Disease Control and Prevention, National Center for Emerging and Zoonotic Infectious Diseases (NCEZID), Division of Healthcare Quality Promotion (DHQP), USA.

Shakya, K.M., Noyes, A., Kallin, R. and Peltier, R.E. (2017). Evaluating the efficacy of cloth facemasks in reducing particulate matter exposure. J. Exposure Sci. Environ. Epidemiol. 27: 352-257.

Spies, A., Wilson, K.S. and Ferrie, R. (2011). Respirator fit of a medium mask on a group of South Africans: a crosssectional study. Environ. Health 10: 17.

Viraneva, A., Yovcheva, T., Krezhov, K. and Sotirov, S. (2015). Electret stability of gamma irradiated PP and PET films. Bulg. Chem. Commun. 47B: 121-126.

Viscusi, D.J., Bergman, M., Sinkule, E. and Shaffer, R.E. (2009). Evaluation of the filtration performance of 21 N95 filtering face piece N95FFRs after prolonged storage. $\mathrm{Am}$. J. Infect Control 37: 381-386.

World Health Organization (2004) Laboratory biosafety manual. World Health Organization, Geneva, Switzerland.

Received for review, January 1, 2020

Revised, March 21, 2020

Accepted, March 21, 2020 